\title{
TRANSIENT PHOTOREFLECTANCE OF DMS: THERMO-MAGNETIC MODULATIONS AND MAGNETIC RELAXATION
}

\section{SCALBert}

Groupe d'Etude des Semiconducteurs, cc074, UMR 5650 CNRS

Université Montpellier 2, Place Eugène Bataillon, 34095 Montpellier Cedex, France

Optical pump-and-probe experiments performed at the nanosecond timescale on $\mathrm{Cd}_{1-x} \mathrm{Mn}_{x} \mathrm{Te}$ are reviewed. Recent studies have established that for low excitation densities thermo-magnetic modulations of the reflectivity prevail against other possible modulations. Hence the relaxation time of the signal directly reflects the spin relaxation of the Mn ions. Detailed studies of the modulation mechanisms give the opportunity to investigate some fundamental magneto-optical properties of the diluted magnetic semiconductors, otherwise beyond reach, such as the paramagnetic shift of the gap in the dilute regime.

PACS numbers: 78.20.Ls, 75.50.Pp, 78.47.+p

\section{Introduction}

Diluted magnetic semiconductors (DMSs) form a broad family of semiconducting materials which are at the frontier between semiconductor physics and magnetism, the most popular ones being the II-VI compounds containing $\mathrm{Mn}^{++}$ ions. They have been intensively studied since the end of the seventies [1]. Their most remarkable properties, such as giant magneto-optical splittings of band edges or magnetic polarons formation, result from the strong exchange interactions between carriers and magnetic ions.

In this paper recent results obtained on CdMnTe will be discussed, one of the best known DMS, by transient reflectivity (TR) and transient photoreflectance (TPR) with about ten nanoseconds resolution. These experiments belong to the family of pump-and-probe experiments which have been developed rapidly during the past two decades. However they differ in the timescales of interest. Indeed most of the pump-and-probe experiments are devoted to the carrier dynamics, especially spin and momentum relaxation, which occurs in the picosecond or sub-picosecond timescale. In DMSs, however, the carriers interact with the local spins of the Mn-ions, the relaxation time of which is much longer due to the weak coupling of these $S$-state ions with the lattice. This fact introduces another timescale in 
the relaxation phenomena of the DMSs. Depending on the Mn-content and on the temperature this relaxation time can vary from the millisecond, or even longer, down to the nanosecond range. Hence one is generally unable to monitor in the same experiment (and in the same experimental conditions) the kinetics of the carriers and that of the Mn spins. A full understanding of the relaxation phenomena in DMSs clearly require a specific experimental setup for probing the above nanosecond regime involved in the local spin relaxation and how this relaxation could be influenced by the photo-injected free carriers.

For this purpose we have performed, in Montpellier, original pump-and-probe experiments with a time resolution of $10 \mathrm{~ns}$, and with the ability to monitor also the relaxation during the pump pulse.

The general idea of these experiments is to follow the Mn-spin relaxation through its influence on the energy of excitonic optical transitions. Indeed, on one hand the energy of these transitions is very sensitive to the magnetization of the sample due to the strong $s-d$ and $p-d$ exchange interactions, and on the other hand the relaxation times of the electron- and hole-spins are much shorter than that of the Mn-spins, therefore one can safely assume that they follow the magnetic relaxation adiabatically.

It will be shown in the following that the results obtained in TR and TPR experiments confirm this simple idea and open up the possibility to monitor the magnetic relaxation at the intermediate timescales in an all-optical experiment.

It is worth mentioning another approach proposed by Strutz et al. who used a pick-up coil for detecting the magnetization changes induced by a laser pulse [2]. Kulakovskii et al. have also performed original pump-probe luminescence experiments with short laser pulses and high excitation densities [3]. Their results suggest the appearance of spatial inhomogeneities in the $\mathrm{Mn}$-spin temperature in the process of heat transfer from the electron-hole plasma to the Mn-spins.

TR and TPR are similar methods because they are both probing changes of the reflectivity induced by the pump beam. TPR however is much more sensitive than TR because it makes use of a modulation technique. Besides the study of magnetic relaxation, much attention has been paid to the modulation mechanisms underlying the TPR signal, providing unexpected results like the possibility to study the magnetic red shift of the gap in the very dilute limit.

In the next section it will be explained how the experimental setup differs from usual pump-and-probe experiments. In Sec. 3 the results obtained in TR experiments will be presented. TPR experiments will be more thoroughly discussed in Sec. 4 before coming to a conclusion.

\section{Experimental details}

The pump-probe setup used to record TPR mainly differs from the conventional pump-and-probe experiments by the use of continuous laser sources chopped by synchronized acousto-optic modulators (AOM) instead of pulsed lasers. The typical rise time of the pulses provided by an AOM is about $10 \mathrm{~ns}$ which sets a lower limit for the time resolution of the experiment. The width of the pulses, the repetition rate, and the relative delay between pump and probe pulses can be varied in a very flexible way. Generally the pump beam was provided by a 
cw-argon laser, and the probe beam was provided by a tunable $\mathrm{Al}_{2} \mathrm{O}_{3}$ :Ti laser. In some experiments a DCM dye laser has been used.

The pump beam is additionally chopped at low frequency and phase sensitive detection gives the modulated signal $\Delta R$.

In the experiments described below bulk $\mathrm{Cd}_{1-x} \mathrm{Mn}_{x}$ Te samples as well as thin layers have been studied. The experiments were done in superfluid helium. The experiments with an external magnetic field were performed in Faraday geometry with $\sigma^{+}$or $\sigma^{-}$polarization of the probe, whereas the pump beam was linearly polarized.

\section{Transient reflectivity}

Transient reflectivity could be measured with the setup described above provided one simply removes the low-frequency modulation of the pump beam and measures the dc-output of the photo-detector for each delay between the pump and probe pulses. Another method has been proposed by Farah et al. [4]. In this method a cw-probe beam was employed and the reflectivity was analyzed by a photo-multiplier and time-resolved in the single-photon counting mode. Acquisition is less time consuming with this setup and enables the acquisition of reflectivity both in the time and in the spectral domains.

The resulting 3D spectra under magnetic field (Fig. 1) revealed large time dependent energy shifts of exciton lines connected with the variations of the spin temperature. For each delay the effective spin temperature can be easily deduced

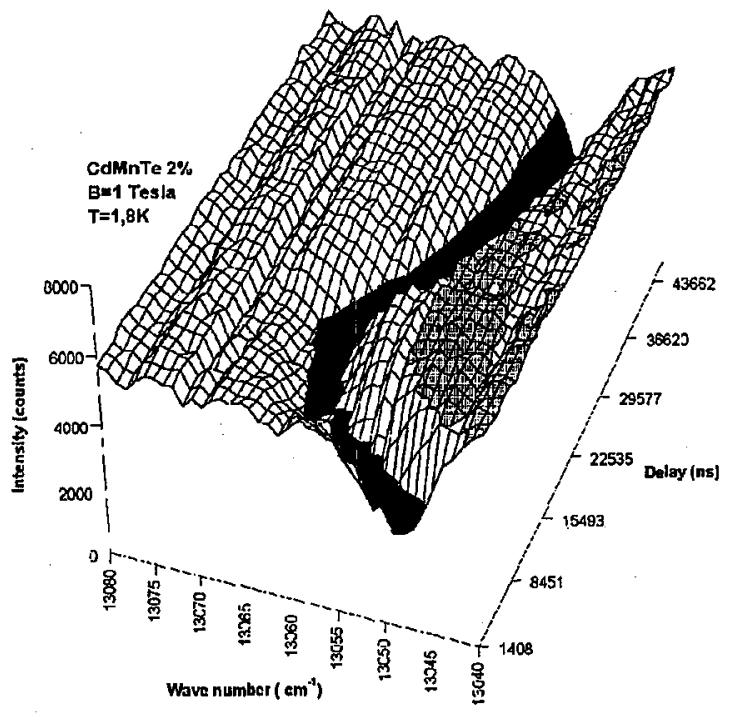

Fig. 1. $3 \mathrm{D}$ spectrum of transient reflectivity versus delay and wave number probed by $\sigma^{+}$-polarized $\mathrm{Ti}: \mathrm{Al}_{2} \mathrm{O}_{3}$ laser. The reflectivity dip observed corresponds to the lowest exciton component (from Ref. [4]). 


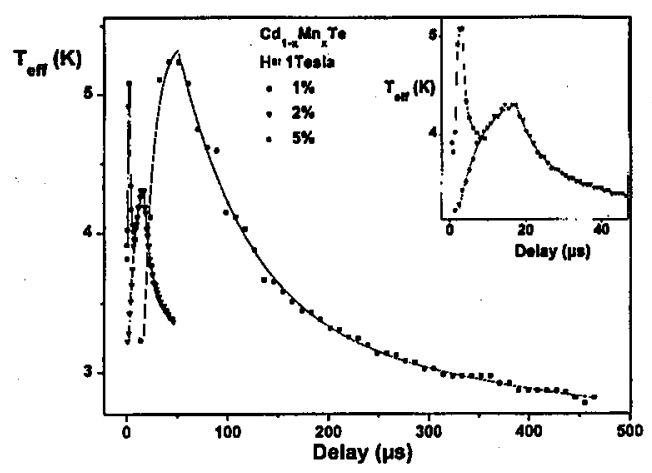

Fig. 2. Typical time evolution of the effective spin temperature as deduced from the time dependent energy shifts of the reflectivity structure recorded in $\sigma^{+}$polarization for different $\mathrm{Mn}^{++}$concentrations. Continuous lines represent one-exponential fits of experimental data during and after the pump pulse (inset: the same curves for 0.05 and 0.02 samples at shorter timescale) (from Ref. [4]).

from the shift by inverting the well-known modified Brillouin function [5]. The time evolution of the spin temperature, as shown in Fig. 2, is faster for increasing Mn concentration a behavior which is characteristic of spin-lattice relaxation.

The relaxation during the pump pulse was faster than after it, which was attributed to a local heating of the lattice during the pump pulse. The influence of photo-carriers on magnetic relaxation is probably negligible at the low excitation density used in these experiments. However at high excitation density an opposite situation can be found [3].

\section{Transient photoreflectance}

Transient photoreflectance being a modulation technique is much more sensitive than transient reflectivity and is well suited to detect small variations of the reflectivity of the sample. We will show below that this ability opens new possibilities which go beyond the study of the magnetic relaxation only.

\subsection{Thermo-magnetic modulations}

Early studies have discussed in detail the possible photo-modulation mechanisms of the reflectivity in the non-magnetic semiconductors and came to the conclusion that the changes in reflectivity are caused by the photo-induced changes in the built-in electric field [6].

Scalbert et al. [7] have shown that in DMSs specific modulations must exist owing to the giant magneto-optical properties of these compounds. Two modulation mechanisms of thermo-magnetic character have been identified: the modulation of the magnetic red shift of the gap (also called paramagnetic shift), and the modulation of the effective $g$-factors (i.e. modulation of the giant Zeeman splittings). Experiments done on thin layers, discussed in Sec. 4.4, also suggest a modulation of the oscillator strength due to the influence of magnetic fluctuations on the effective mass of the carriers. 


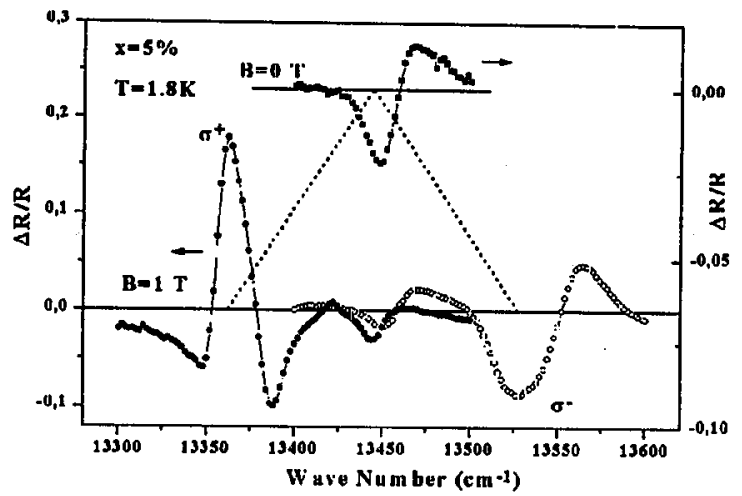

Fig. 3. Transient photoreflectance spectra of $\mathrm{Cd}_{0.95} \mathrm{Mn}_{0.05} \mathrm{Te}$ for $B=0$ and $B=1 \mathrm{~T}$ in Faraday configuration recorded at a fixed delay (from Ref. [7]).

Figure 3 gives typical TPR spectra of $\mathrm{Cd}_{0.95} \mathrm{Mn}_{0.05} \mathrm{Te}$ recorded for a given delay of the probe pulse with respect to the pump pulse, in Faraday geometry for $B=0$ and $B=1$ tesla, and for $\sigma^{+}$and $\sigma^{-}$polarizations of the probe. The dotted lines represent the giant Zeeman splitting of the strong exciton components.

In a magnetic field of 1 tesla the $\sigma^{+}$and $\sigma^{-}$strong components, corresponding to $|+3 / 2,-1 / 2\rangle$ and $|-3 / 2,+1 / 2\rangle$ excitons, have opposite signs. This is the signature of a spin-dependent modulation, namely the thermal modulation of the giant Zeeman splitting. The situation is different for the weak exciton components which have the same sign excluding the preceding modulation as being the dominant one. This is not surprising because of the much smaller Zeeman splitting in this case.

The zero-field signal has been attributed to the photo-modulation of the paramagnetic shift of the gap, mainly on the basis of its relaxation time which co-

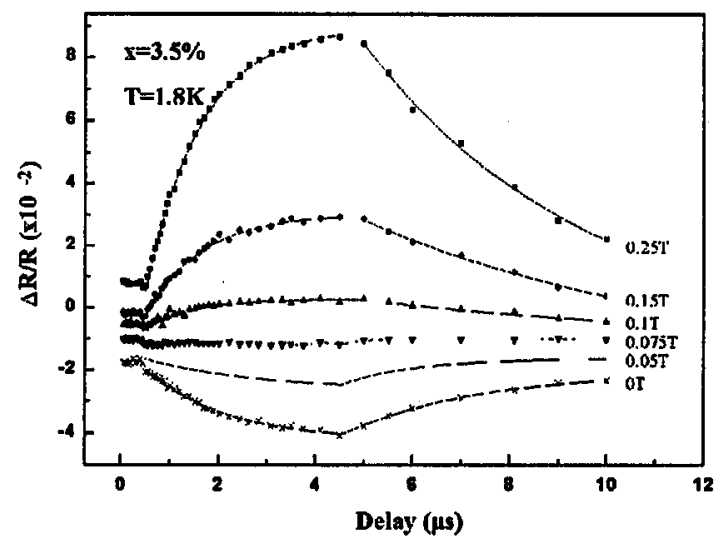

Fig. 4. Time dependence of photoreflectance for different magnetic fields recorded at the peak of TPR spectrum in $\sigma^{+}$polarization. Solid lines are exponential fits to the data (from Ref. [7]). 
incides with that of the field-induced signal (Fig. 4). The negative sign in zero-field corresponds to a red shift during illumination as expected for this modulation. The striking field-induced change of the sign of the photoreflectance signal for $\sigma^{+}$polarization of the probe will serve as a new method for investigating the paramagnetic shift at low concentrations (cf. Sec. 4.3).

To sum up, the analysis of TPR experiments performed on CdMnTe has revealed two new modulation mechanisms of thermo-magnetic character: modulation of the Zeeman splitting and modulation of the paramagnetic shift of the gap.

\subsection{Relaxation rates}

One of the interesting issues in these relaxation measurements is to seek for an eventual influence of the photo-injected carriers on the relaxation rate of the Mn-spins. The pump-and-probe experiments using acousto-optic modulators are well adapted for this purpose because the width of the pump pulses can be set at any desired value compatible with the relaxation times involved. Hence one can measure in a single experiment the relaxation time during the pump pulse, in presence of photo-carriers, and after it, when carriers have recombined, keeping in mind that the lifetime of the carriers is much shorter than the spin relaxation time of manganese.

The results reported in Ref. [7] and shown in Fig. 5 revealed a strong increase in the relaxation rates, in zero field, during the pump pulse and with increasing pump intensity, while they remain practically constant after the pump. One could be tempted to attribute this more rapid relaxation during the pump to the presence of photo-carriers. However a heating of the lattice during the pump will produce a similar effect because the spin-lattice relaxation is faster when temperature increases.

We will see in Sec. 4.4 that experiments on thin layers settle in favor of the thermal effect.

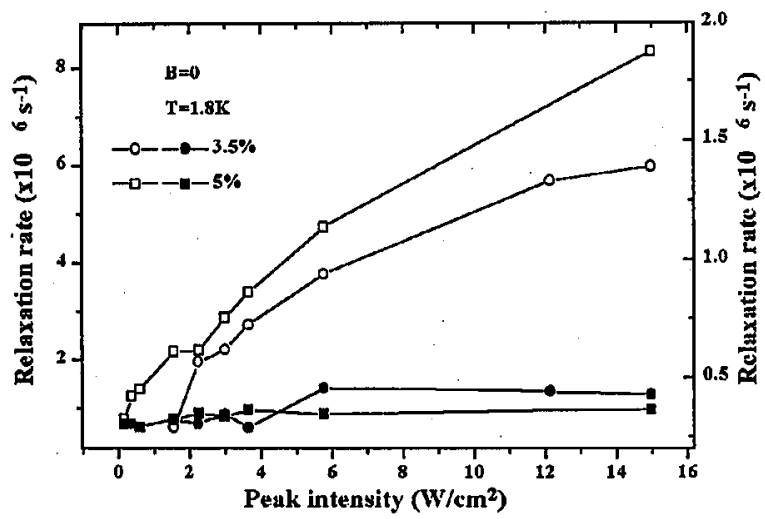

Fig. 5. Pump intensity dependence of relaxation rates during (open symbols) and after the pump pulse (solid symbols) for $x=5 \%$ (left axis) and 3.5\% (right) (from Ref. [7]). 


\subsection{Study of the paramagnetic shift in the dilute regime}

In Sec. 4.1 we have described the two thermo-magnetic modulations which are dominant in the photoreflectance of CdMnTe at low temperature. The field-induced change of sign of the signal observed in Faraday configuration and for the right-handed circular polarization of the probe can be explained as a result of the competition between these two modulations.

In $\sigma^{+}$polarization the paramagnetic shift modulation and the $g$-factors modulation have opposite signs. Since the $g$-factors modulation increases with the magnetic field there exists a particular field $B_{\mathrm{c}}$ for which these two energy modulations cancel each other. As a consequence, the sign of TPR changes when the magnetic field crosses $B_{\mathrm{c}}$.

The determination of $B_{\mathrm{c}}$ has been proposed in Ref. [8] as a new method for investigating the paramagnetic shift, and because of the great sensitivity of this method it was possible to extend these studies to concentrations much lower than $10 \%$ which was the lowest investigated concentration before.

If we denote $\Delta E_{\mathbf{P S}}$ the amplitude of the paramagnetic shift modulation and $\Delta E_{\mathrm{Z}}$ that of the Zeeman shift modulation for the appropriate strong exciton line, $B_{\mathrm{c}}$ corresponds to $\Delta E_{\mathrm{PS}}+\Delta E_{\mathrm{Z}}=0$. For thermal modulations we have $\Delta E=(\partial E / \partial T) \Delta T$, where $\Delta T$ represents the temperature modulation. Hence at the crossing-field $\left(\partial E_{\mathrm{PS}} / \partial T\right)=-\left(\partial E_{\mathrm{Z}} / \partial T\right)_{B=B_{\mathrm{c}}}$. This equality constitutes the central idea of the method proposed in Ref. [8]. The right-hand term of the equality being well known, once $B_{\mathrm{c}}$ is measured one gets the temperature derivative of the paramagnetic shift.

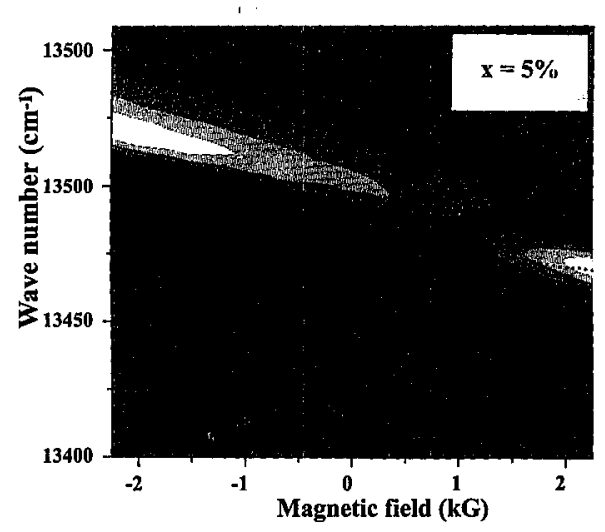

Fig. 6. Contour plot of transient photoreflectance $\Delta R / R$ in the plane (wave number, magnetic field) recorded at $2 \mathrm{~K}$ in Faraday geometry and at a constant delay. Dark (resp. bright) areas correspond to negative (resp. positive) signal. The levels are equally spaced $\left(1.5 \times 10^{-3}\right)$. Positive (resp. negative) fields correspond to $\sigma^{+}$(resp. $\sigma^{-}$) polarization and the overall slope is due to the Zeeman shift. The dotted curve shows the position of the excitonic resonance (from Ref. [8]). 


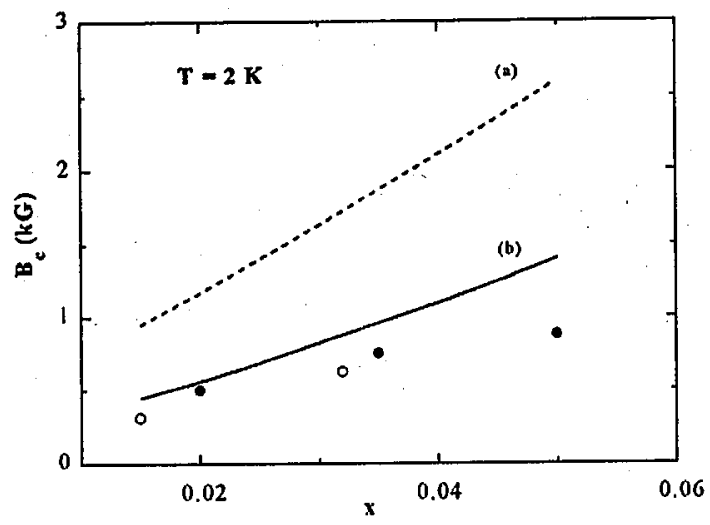

Fig. 7. Crossing-field $B_{\mathrm{c}}$ versus molar fraction of $\mathrm{Mn}^{++}$content. The dashed and solid lines are theoretical values calculated in the approximation where interference effects are neglected and included, respectively. The circles correspond to experimental values measured on $\mathrm{Cd}_{1-x} \mathrm{Mn}_{x}$ Te layers (open circles) and bulk samples (close circles) (from Ref. [8]).

The sign-reversal of the photoreflectance is illustrated in Fig. 6 where a contour plot of $\Delta R / R$ is represented, for a fixed delay, as a function of wave number and magnetic field. The arrow indicates the crossing-field $B_{\mathrm{c}}$. However for a precise determination of $B_{\mathrm{c}}$ time-resolved measurements were used. In particular, it makes possible to separate the magnetic contributions from the non-magnetic ones, whose relative contribution increases with the dilution.

The results (see Fig. 7) indicated a pronounced reduction of $B_{\mathrm{c}}$, and consecutively of the paramagnetic shift, compared to the prediction of the macroscopic model proposed by Bylsma et al. [9] and by Gaj and Golnik [10]. This reduction has been attributed in Ref. [5] to the interference between Bloch waves scattered by spin-correlated ions. These interferences are partly destructive and their effect becomes more crucial at low temperature when long-range spin-spin correlations develop.

\subsection{Optical path modulation in thin layers}

In Ref. [11] Farah et al have introduced the optical path modulation (OPM), an interferometric photoreflectance method, based on a variation of the refractive index of a DMS layer forming a Fabry-Perot resonator. The refractive index is influenced by magnetic fluctuations of the layer and its magnetization induced by an external magnetic field.

Figure 8 shows an example of transient photoreflectance spectrum obtained on. a $3 \mu \mathrm{m}$ thick $\mathrm{Cd}_{0.97} \mathrm{Mn}_{0.03}$ Te MBE layer. Line $X$ is the excitonic line, and the structures $A, B, C$ are related to the OPM. Their derivative-like lineshape proves that they result from a modulation of the optical thickness of the layer. This is possible if a non-vanishing modulation of the refractive index averaged over the layer thickness exists. The OPM can thus be considered as a volume effect. If the 


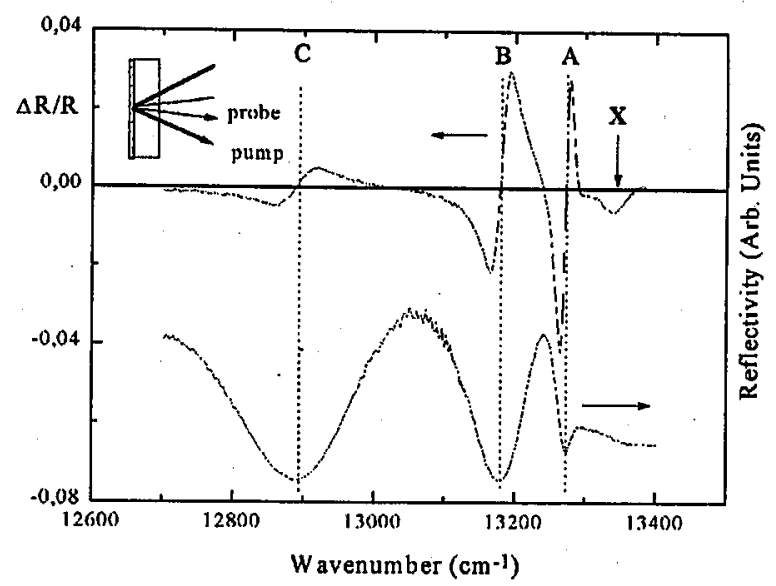

Fig. 8. Reflectivity and transient photoreflectance (TPR) spectra of a $3 \mu \mathrm{m}$ thick layer of $\mathrm{Cd}_{0.97} \mathrm{Mn}_{0.03}$ Te glued on a glass plate and immersed in superfluid helium $(T=2 \mathrm{~K})$, at zero-magnetic field. The TPR spectrum is measured for probe pulses set at the end of the pump pulses (inset shows the pump-probe configuration) (from Ref. [11]).

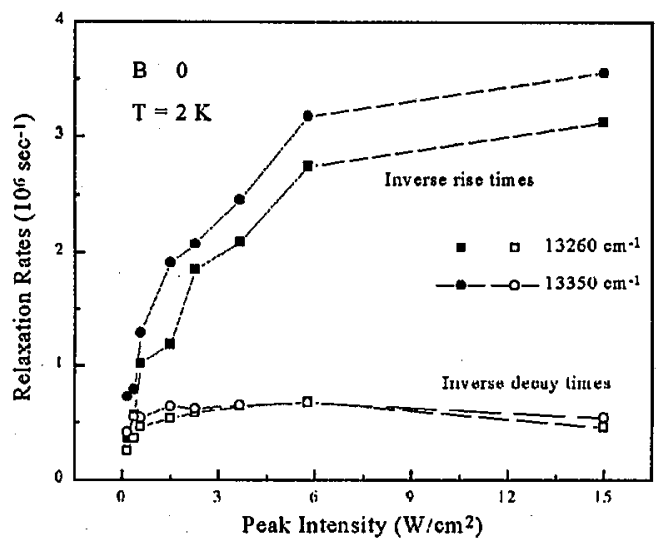

Fig. 9. Relaxation rates measured at two probe energies corresponding to free exciton line (relaxation of near surface $\mathrm{Mn}^{++}$ions is measured) and to $A$ structure (average relaxation of all $\mathrm{Mn}^{++}$is measured) in configuration of Fig. 8. Closed symbols: inverse rise time (during the pump pulse), open symbols: inverse decay time (after the pump pulse) (from Ref. [11]).

probe-wavelength is tuned to one of the OPM structure the transient photoreflectance yields an information averaged on Mn spins distributed inside the layer, whereas probing line $X$ yields information on $\mathrm{Mn}$ spins near the surface due to the strong optical absorption at the corresponding energy. This makes possible to discriminate between the thermal-induced or carrier-induced speeding up of the magnetic relaxation rate observed for increasing pump intensity in bulk samples (see Sec. 4.2). The comparison between the relaxation rates measured with 


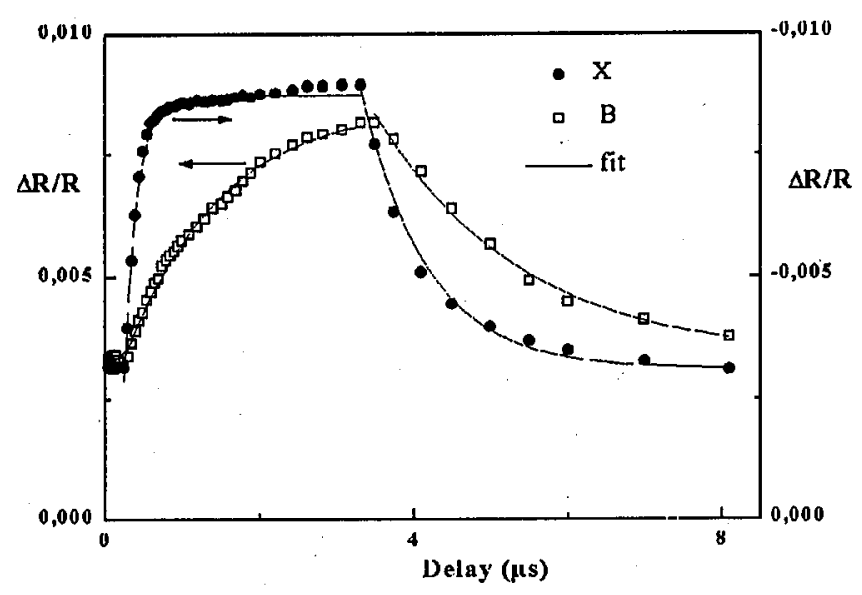

Fig. 10. Temporal dependence of the photoreflectance for probe energies corresponding to the exciton line $X\left(14870 \mathrm{~cm}^{-1}\right)$ and to the OPM line $B\left(14725 \mathrm{~cm}^{-1}\right)$ for $\mathrm{Ar}^{++}$ excitation (lines give exponential fits of the data) (from Ref. [12]).

surface-probing (line $X$ ) and with volume-probing (line $A$ ) have revealed a very similar behavior as a function of pump intensity (Fig. 9) which excludes an influence of carriers whose distribution normal to the surface is strongly non-uniform.

A quite different behavior has been reported in Ref. [12] for much concentrated CdMnTe layers containing $16 \%$ molar fraction of $\mathrm{Mn}$ ions (Fig. 10). In this case volume probing gives a much longer relaxation time than surface probing. It seems unlikely that this difference could be due to the injected carriers, because the difference in the relaxation times persists after the pump pulse when carriers have recombined. Also the relaxation time measured with volume-probing seems anormally long for the concentration considered. Probably the temporal behavior in this case is governed by heat diffusion inside the layer, which indicates a dramatic slowing down of the diffusion in this concentrated sample. Akimov et al. [13] have reported a method based on luminescence for detecting nonequilibrium acoustic phonons in DMSs heterostructures which could be also helpful for measuring the heat diffusion.

The OPM structures also exhibit a field-induced sign reversal for $\sigma^{+}$-probe polarization [11], but surprisingly the sign reversal is not simultaneous for the three structures observed. On the contrary, it occurs according to a definite sequence with increasing field, which could be explained only by invoking the existence of an oscillator strength modulation. It has been tentatively attributed to a manifestation of the influence of magnetic fluctuations on the effective mass of carriers.

\section{Conclusion}

An overview of recent pump-probe optical experiments done in the nanosecond time domain has been presented, which give access to the spin relaxation of $\mathrm{Mn}^{++}$in CdMnTe. It has been tried to demonstrate in this review that these experiments are also very promising for studying some fine magneto-optical properties of DMSs such as the paramagnetic shift of the gap in the dilute limit. 


\section{Acknowledgments}

The author would like to thank his co-workers who contributed to different parts of the studies presented in this manuscript: W. Farah, M. Nawrocki, J.A. Gaj, Y.G. Semenov, E. Janik, G. Karczewski, and T. Wojtowicz.

\section{References}

[1] Diluted Magnetic Semiconductors, Eds. J.K. Furdyna, J. Kossut, in series Semiconductors and Semimetals, Vol. 25, Academic Press, Boston 1988; Semimagnetic Semiconductors and Diluted Magnetic Semiconductors, Eds. M. Averous, M. Balkanski, Plenum, London 1991.

[2] T. Strutz, A.M. Witowski, P. Wyder, Phys. Rev. Lett. 26, 3912 (1992); Rev. Sci. Instrum. 64, 1853 (1993).

[3] V.D. Kulakovskii; M.G. Tyazhlov, A.I. Filin, D.R. Yakovlev, A. Waag, G. Landwehr, Phys. Rev. B 54, R8333 (1996); M.G. Tyazhlov, A.I. Filin, A.V. Larionov, V.D. Kulakovskii, D.R. Yakovlev, A. Waag, G. Landwehr, JETP 85, 784 (1997) [Zh. Eksp. Teor. Fiz. 112, 1440 (1997)].

[4] W. Farah, D. Scalbert, M. Nawrocki, Phys. Rev. B 53, R10461 (1996).

[5] J.A. Gaj, R. Planel, G. Fỉshman, Solid State Commun. 29, 435 (1979).

[6] Modulation Spectroscopy, Solid State Physics, Ed. M. Cardona, Academic Press, New York 1969; Modulation Techniques, Eds. R.K. Willardson, A.C. Beer, in series Semiconductors and Semimetals, Vol. 9, Academic, New York 1972.

[7] D. Scalbert, W. Farah, M. Nawrocki, in: Proc. 23rd Int. Conf. Phys. Semicond., Eds. M. Scheffler, R. Zimmermann, World Scientific, Singapore 1996, p. 433.

[8] W. Farah, D. Scalbert, M. Nawrocki, Y.G. Semenov, to appear in Phys. Rev. B.

[9] R.B. Bylsma, W.M. Becker, J. Kossut, U. Debska, D. Yoder-Short, Phys. Rev. B 33, 8207 (1986).

[10] J.A. Gaj, A. Golnik, Acta Phys. Pol. A 71, 197 (1987).

[11] W. Farah, D. Scalbert, M. Nawrocki, J.A. Gaj, E. Janik, G. Karczewski, T. Wojtowicz, Phys. Rev. B 57, 8770 (1998).

[12] W. Farah, D. Scalbert, M. Nawrocki, J.A. Gaj, E. Janik, G. Karczewski, T. Wojtowicz, J. Cryst. Growth 184/185, 980 (1998).

[13] A.V. Akimov, A.V. Scherbakov, A.L. Zhmodikov, V.P. Kochereshko, D.R. Yakovlev, W. Ossau, G. Landwehr, T. Wojtowicz, G. Karczewski, J. Kossut, Phys. Rev. B 56, 12100 (1997). 